\title{
Effective combination of aligned nanocomposite nanofibers and human unrestricted somatic stem cells for bone tissue engineering
}

\author{
Behnaz BAKHSHANDEH ${ }^{1,2}$, Masoud SOLEIMANI ${ }^{3, *}$, Nasser GHAEMI ${ }^{4}$, Iman SHABANI ${ }^{2}$ \\ ${ }^{1}$ Department of Biotechnology, College of Science, University of Tehran, Tehran, Iran; ${ }^{2}$ Stem Cell Biology Department, Stem Cell Tech- \\ nology Research Center, Tehran, Iran; ${ }^{3}$ Department of Hematology, Faculty of Medical Sciences, Tarbiat Modares University, Tehran, \\ Iran; ${ }^{4}$ School of Chemistry, College of Science, University of Tehran, Tehran, Iran
}

Aim: Bioartificial bone tissue engineering is an increasingly popular technique to solve bone defect challenges. This study aimed to investigate the interactions between matrix composition and appropriate cell type, focusing on hydroxyapatite (HA), to achieve a more effective combination for bone regeneration.

Methods: Human unrestricted somatic stem cells (USSCS) were isolated from placental cord blood. The cellular and molecular events during the osteo-induction of USSCs were evaluated for $21 \mathrm{~d}$ under the following conditions: (1) in basal culture, (2) supplemented with hydroxyapatite nanoparticle ( $\mathrm{nHA}$ ) suspension, and (3) seeded on electrospun aligned nanofibrous poly- $\varepsilon$-caprolactone/poly-L-lactic acid/nHA (PCL/PLLA/nHA) scaffolds. The scaffolds were characterized using scanning electron microscope (SEM), fourier transform infrared spectroscopy (FTIR) and tensile test.

Results: Maintenance of USSCs for $21 \mathrm{~d}$ in basal or osteogenic culture resulted in significant increase in osteoblast differentiation. With nHA suspension, even soluble osteo-inductive additives were ineffective, probably due to induced apoptosis of the cells. In contrast to the hindrance of proliferation by $\mathrm{nHA}$ suspension, the scaffolds improved cell growth. The scaffolds mimic the nanostructure of natural bone matrix with the combination of PLLA/PCL (organic phase) and HA (inorganic phase) offering a favorable surface topography, which was demonstrated to possess suitable properties for supporting USSCs. Quantitative measurement of osteogenic markers, enzymatic activity and mineralization indicated that the scaffolds did not disturb, but enhanced the osteogenic potential of USSCs. Moreover, the alignment of the fibers led to cell orientation during cell growth.

Conclusion: The results demonstrated the synergism of PCL/PLLA/nHA nanofibrous scaffolds and USSCs in the augmentation of osteogenic differentiation. Thus, nHA grafted into PCL/PLLA scaffolds can be a suitable choice for bone tissue regeneration.

Keywords: human unrestricted somatic stem cells; electrospun aligned nanofibrous poly-e-caprolactone/poly-L-lactic acid/nHA scaffolds; bone regeneration; tissue engineering

Acta Pharmacologica Sinica (2011) 32: 626-636; doi: 10.1038/aps.2011.8; published online 25 Apr 2011

\section{Introduction}

Bone defects caused by injury or disease are one of the major health concerns of human society. Current practice mainly relies on tissue repair by autograft and allograft or application of synthetic bone implants ${ }^{[1,2]}$. However, many limitations, such as donor morbidity, limited availability of tissue suppliers, potent immune response, disease transfer, or insufficient bioactivity, have led this field to tissue engineering as an excellent alternative approach ${ }^{[3-7]}$.

\footnotetext{
* To whom correspondence should be addressed.

E-mail soleim_m@modares.ac.ir

Received 2010-08-03 Accepted 2011-01-18
}

Cell transplantation in combination with supportive matrices for re-establishment of altered constructs is emerging as a new strategy for bone tissue engineering ${ }^{[2,8,9]}$. Unique features such as high porosity with proper interconnections, large surface area and enhanced recruiting of cells make nanofibrous scaffolds suitable for tissue engineering ${ }^{[10,11]}$. Because of the importance of nano-sized structures of the extracellular matrix (ECM) in the regulation of many cell functions, scaffolds with nanoscale morphology have been vigorously studied ${ }^{[9,10]}$. In this regard, developing nanofibrous scaffolds that mimic the architecture of tissue at the nanoscale can potentially meet this challenge $^{[12]}$. Irrespective of the method of synthesis, nanofibers have been used as scaffolds for musculoskeletal tissue engineering (including bone, cartilage, ligament, and skeletal 
muscle), vascular tissue engineering, and skin tissue engineering, as well as carriers for proteins and $\mathrm{DNA}^{[2,4]}$.

Electrospinning is one of the most broadly considered techniques to process polymeric biomaterials into nanofibers. Control over the thickness and composition of the porous nanofibers is an important aspect of this technique ${ }^{[10]}$.

Hydroxyapatite (HA) composites have received a great deal of attention for bone regeneration because they possess excellent biodegradability, biocompatibility, osteoconductivity and direct bone-binding capability ${ }^{[1,3]}$. Yang et a ${ }^{[13]}$ demonstrated that the incorporation of HA into PCL/gelatin nanofibers enhanced dental pulp stem cell differentiation toward an odontoblast-like phenotype in vitro and in vivo. Osteogenic induction properties of HA has also been proven using goat and rat MSCs ${ }^{[14,15]}$. Finally, it has been shown that incorporation of HA nanofibers as a second component in $\beta$-tricalcium phosphate significantly improves the mechanical strength of composite scaffolds ${ }^{[16]}$.

However, owing to brittleness and poor mechanical stability, the use of HA without additional ingredients is limited ${ }^{[17]}$.

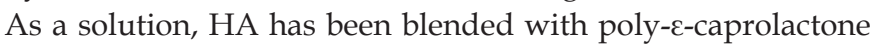
(PCL) and poly-L-lactic acid (PLLA). To overcome the possible masking of hydroxyapatite nanoparticle (nHA) osteoinductive properties due to it being embedded inside the polymer, the electrospun nanofiber technique has been strongly suggested $^{[11]}$.

PCL is a synthetic, biodegradable polymer with a slow degradation rate ${ }^{[5]}$. It has been used in fields such as skin tissue engineering and axonal regeneration and as a support for fibroblast and osteoblast growth ${ }^{[18]}$. It has been reported that adhesion and proliferation of fibroblasts and protein adsorption are improved in PCL/HA scaffolds compared to unmodified HA and pure PCL controls ${ }^{[19]}$. Yoshimoto $e t$ al ${ }^{[20]}$ have reported that mesenchymal stem cells (MSCs) seeded on electrospun PCL scaffolds migrated inside the scaffold and produced a rich extracellular matrix. Shin et al ${ }^{[21]}$ replicated this experiment in a rat model and demonstrated ECM production all over the scaffold, along with mineralization and osteogenic differentiation.

PLLA, the L-form of poly-lactic acid (PLA), is an FDAapproved polyester that has been studied extensively as a biodegradable surgical material; however, because of its poor biocompatibility and mechanical properties, its use is limited ${ }^{[8,22]}$. The PLLA/HA nanocomposite improves the interface mechanical property and biocompatibility, and it is applied widely for bone fixation materials ${ }^{[23]}$. Also, incorporation of HA into PLLA enhances protein adsorption and increases the compressive modulus in comparison with pure PLLA scaffolds ${ }^{[24]}$. In one study, mouse fibroblasts cultured on PLLA/HA scaffolds showed a reduction in the inflammatory response compared with pure PLLA ${ }^{[25]}$.

The stress-shielding problem is a result of elastic modulus mismatch between metallic materials and natural bone. Consequently, creation of polymer-ceramic composites that match the modulus of bone more closely has been suggested as a solution $^{[26]}$. In one study, researchers successfully produced
PLLA/HA/PCL composites with flexural moduli near the lower range of bone ${ }^{[27]}$. Hence, various nanofibers of chitosan/ HA, PCL/collagen/HA, PCL/gel/HA, gel/HA, PCL/HA/ gel, PCL/HA, and PHBV/HA have been generated for bone tissue engineering ${ }^{[28-32]}$.

In this study, both the cell type that was loaded and the scaffold composition were important factors to consider when attempting to achieve a more effective combination for bone tissue regeneration. In previous reports on bone tissue engineering, hMSCs, preosteoblast cell lines and human osteoblasts have been routinely cultured. In contrast to adult bone marrow, human unrestricted somatic stem cells (USSCs) are less mature, with an extended length of life and longer telomeres. In addition to these biological advantages, USSCs are abundantly available and routinely harvested without risk to the donor ${ }^{[33-35]}$.

This adherent HLA class II and CD45-negative stem cell can be stimulated to differentiate into bone, cartilage, hematopoietic cells, neural cells, liver and heart ${ }^{[2,34]}$. It is worth noting that spontaneous differentiation of USSCs into osteoblasts has been demonstrated ${ }^{[36]}$. As a result, a USSC-based tissue engineering strategy seems to be an ideal approach for bone regeneration. However, the effect of nHA-containing nanofibers on differentiation and mineralization of USSCs has yet to be investigated.

We electrospun blends of nHA with both PCL and PLLA to create an in vitro environment that resembles the lowest level of hierarchical organization of bone for tissue engineering. The dispersion and effects of nHA on the tensile and morphological properties of nanofibers were investigated. In addition, the effects of PLLA/PCL/nHA nanofibers or an nHA suspension on the proliferation and osteogenic differentiation of USSCs were assessed.

\section{Materials and methods \\ Materials}

PCL $\left(M_{\mathrm{n}}=80\right)$, PLLA $\left(M_{\mathrm{n}}=99\right)$, and nHA were obtained from Sigma-Aldrich. The solvents $\mathrm{N}, \mathrm{N}$-dimethylformamide (DMF) and chloroform were purchased from Merck. All other chemicals were purchased from Sigma Co (St Louis, MO, USA) and used as received, unless stated otherwise.

\section{Cell seeding}

Collection, isolation and propagation of human USSCs were performed as described by Kogler et $\mathrm{al}^{[34]}$. Growth and expansion of the cells were performed in high glucose Dulbecco's modified Eagle's medium (DMEM) supplemented with 10\% fetal bovine serum and $1 \%$ antibiotic $(100 \mathrm{U} / \mathrm{mL}$ penicillin) under a humidified atmosphere of $95 \%$ air with $5 \% \mathrm{CO}_{2}$ at $37^{\circ} \mathrm{C}$. USSCs were cultured in 3 groups (6 subgroups) as described in Table 1. Five duplicate wells were prepared for each sample. Prior to cell seeding, scaffolds were placed in 4-well tissue culture polystyrene (TCPS) and sterilized in $70 \%$ ethanol. The scaffolds were then incubated with basal medium overnight to facilitate cell attachment. An initial cell density of $10^{4} \mathrm{~cm}^{-1}$ was used for all groups. The average size of 
Table 1. Description of different media of USSC cultivation.

\begin{tabular}{cllll}
\hline Group & Subgroup & Matrix & Media & Additional material \\
\hline \multirow{2}{*}{1} & TCPS & 4-well plate & High glucose DMEM, FBS & - \\
& Osteo-TCPS & 4-well plate & Osteo-inductive media & - \\
2 & Osteo-nHA & 4-well plate & Osteo-inductive media & HA nanoparticle suspension \\
\multirow{2}{*}{3} & nHA & 4-well plate & High glucose DMEM, FBS & HA nanoparticle suspension \\
& Scaffold & HA scaffold & High glucose DMEM, FBS & - \\
& Osteo-Scaffold & HA scaffold & Osteo-inductive media & - \\
\hline
\end{tabular}

* tissue culture polystyrene.

the nHA was less than $200 \mathrm{~nm}$. For group 2, after the cells had anchored to the plates, the culture medium was replaced with an nHA suspension $(500 \mathrm{mg} / \mathrm{L})$. The medium was exchanged twice a week.

\section{Fabrication of nanofibrous scaffolds}

We constructed PCL/PLLA and PCL/PLLA/nHA nanofibrous webs by electrospinning. Electrospinning solutions were prepared by dissolving PCL to a concentration of $8 \%$ $(w / w)$ and PLLA to a concentration of $4 \%(w / w)$ in a solvent mixture of chloroform and DMF $(8: 2, v / v)$. For PCL/PLLA/ nHA scaffolds, nHA (20\% of polymer weight $(w / w))$ was dispersed in chloroform to form a suspension, and then the polymer was dissolved in the suspension. Finally, the second solvent (DMF) was added, and the mixture was homogenized by stirrer and ultrasonic homogenizer. A $10 \mathrm{~mL}$ disposable syringe was used to stock each of the prepared solutions. Two syringe pumps were used to feed the solutions through extension tubes capped with blunted 21-gauge needles. The collecting surface consisted of a cylindrical stainless steel collector rotating at 2300 revolutions per minute. The collector was located a fixed distance $(15 \mathrm{~cm})$ from the needle. A high voltage potential $(20 \mathrm{kV})$ was also applied between the needle and the collector. The polymer solution was forced through the needle and collected as nanofibers on the rotating cylinder. The aligned nanofibrous membranes were separated from the collector surface and used for further analysis and application. Oxygen plasma treatment was performed by a low frequency plasma generator of $44 \mathrm{kHz}$ frequency with a cylindrical quartz reactor (Diener Electronics, Germany). Pure oxygen was introduced into the reaction chamber at 0.4 mbar pressure, and then the glow discharge was ignited for $3 \mathrm{~min}$.

\section{Cell and fiber morphology}

For the evaluation of nanofiber morphology, the specimens were gold coated using a sputter coater and then scanned by a scanning electron microscope (SEM, LEO 1455VP, Cambridge, UK). The fiber diameter was determined from SEM images using image analysis software (imageJ, NIH, USA). The cellloaded scaffolds were rinsed with PBS after $14 \mathrm{~d}$ of culture and fixed in $2.5 \%$ glutaraldehyde for $1 \mathrm{~h}$. For dehydration, the cellloaded scaffolds were placed in a gradient series of increasing alcohol concentrations and then dried. Then, as for the pure scaffold, they were gold coated and scanned by SEM.

\section{Mechanical properties}

The tensile properties were measured on the electrospun PCL/ PLLA and PCL/PLLA/nHA nanofibrous webs in the parallel direction using Galdabini testing equipment. Prepared scaffolds were cut into $10 \mathrm{~mm} \times 50 \mathrm{~mm} \times 0.12 \mathrm{~mm}$ specimens and the tensile test was conducted at $20 \mathrm{~mm} / \mathrm{min}$ crosshead speed at room temperature.

\section{ATR-FTIR spectroscopy}

Fourier transform infrared (FTIR) spectroscopy was performed to investigate the incorporation of nHA into the electrospun nanofibers. FTIR spectra were obtained with an Equinox 55 spectrometer (Bruker Optics, Germany) with a resolution of $2 \mathrm{~cm}^{-1}$.

\section{Osteogenic differentiation}

For induction of USSCs into osteoblasts, the growth medium was supplemented with $250 \mathrm{nmol} / \mathrm{L}$ dexamethasone, 50 $\mu \mathrm{g} / \mathrm{mL}$ ascorbic acid 2-phosphate, and $10 \mathrm{mmol} / \mathrm{L}$ beta-glycerol phosphate.

\section{Differential staining}

To determine calcium deposition, cultured cells were treated with alizarin red S. Briefly, samples were rinsed in PBS, fixed with $4 \%$ paraformaldehyde ( $1 \mathrm{~h}$, room temperature), washed with PBS and stained with fresh $1 \%$ alizarin red $\mathrm{S}, \mathrm{pH} 4.1$, for $10 \mathrm{~min}$ at room temperature. Wells were then rinsed with a $0.1 \% \mathrm{HCl}$ solution to remove excess stain and visualized using a light microscope.

\section{Cell proliferation assay}

The total protein content of each sample was determined using the Pierce BCA Protein Assay Kit (Thermo Scientific, USA) according to the manufacturer's instructions. After lysis, centrifugation and incubation of the mixture at $37^{\circ} \mathrm{C}$ for $30 \mathrm{~min}$, absorbance was measured at $562 \mathrm{~nm}$.

\section{Real-time quantitative RT-PCR}

Total cellular RNA was extracted using QIAzol (Qiagen, USA). Synthesis of cDNA was carried out with M-MuLV reverse transcriptase (RT) and random hexamers as primers, accord- 
ing to the manufacturer's instructions (Fermentas).

Reaction mixtures for PCR included $2.5 \mu \mathrm{L}$ cDNA (5 $\mu \mathrm{mol} / \mathrm{L}), 1 \times \mathrm{PCR}$ buffer (AMS ${ }^{\mathrm{TM}}$, Cinnagen, Iran), $200 \mu \mathrm{mol} / \mathrm{L}$ dNTPs, $0.5 \mu \mathrm{mol} / \mathrm{L}$ of both the forward and the reverse primer and $1 \mathrm{U}$ Taq DNA polymerase (Fermentas, MD, USA). PCR amplification was performed using a standard procedure with denaturation at $94{ }^{\circ} \mathrm{C}$ for $15 \mathrm{~s}$, annealing at 53-61 ${ }^{\circ} \mathrm{C}$ (depending on the primers) for $30 \mathrm{~s}$, and extension at $72{ }^{\circ} \mathrm{C}$ for $45 \mathrm{~s}$. The number of cycles varied between 30 and 40, depending on the abundance of the particular mRNA (Table 2). Amplified DNA fragments were electrophoresed on a $2 \%$ agarose gel. The gels were stained with ethidium bromide $(10 \mu \mathrm{g} / \mathrm{mL})$ and photographed on a UV trans-illuminator (UVIdoc, UK).

Table 2. Primers used for RT-PCR and quantitative real-time PCR.

\begin{tabular}{|c|c|c|c|}
\hline Gene & $\begin{array}{c}\text { Primer sequences } \\
\text { Sense, top; antisense, bottom }\end{array}$ & $\begin{array}{l}\mathrm{Tm} \\
\left({ }^{\circ} \mathrm{C}\right)\end{array}$ & $\begin{array}{r}\text { Size } \\
(\mathrm{bp})\end{array}$ \\
\hline Osteonectin & $\begin{array}{l}\text { 5'-AGGTATCTGTGGGAGCTAATC-3' } \\
\text { 5'-ATTGCTGCACACCTTCTC-3' }\end{array}$ & 60 & 223 \\
\hline BMP-2 & $\begin{array}{l}\text { 5'-TGCGGTCTCCTAAAGGTC-3' } \\
\text { 5'-AACTCGAACTCGCTCAGG-3' }\end{array}$ & 60 & 185 \\
\hline ALPase & $\begin{array}{l}\text { 5'-GCACCTGCCTTACTAАCTC-3' } \\
\text { 5'-AGACACCCATCCСАTCTC-3' }\end{array}$ & 48 & 161 \\
\hline HPRT & $\begin{array}{l}\text { 5'-CCTGGCGTCGTGATTAGTG-3' } \\
\text { 5'-TCAGTCCTGTCCATAATTAGTCC-3' }\end{array}$ & 58 & 125 \\
\hline Osteocalcin & $\begin{array}{l}\text { 5'-TCACACTCCTCGCCCTATTGG-3' } \\
\text { 5'-GATGTGGTCAGCCAACTCGTCA-3' }\end{array}$ & 58 & 250 \\
\hline Osteopontin & $\begin{array}{l}\text { 5'-AGTTTCGCAGACCTGACATC-3' } \\
\text { 5'-ACCATTCAACTCCTCGCTTTC-3' }\end{array}$ & 54.6 & 82 \\
\hline
\end{tabular}

For real-time PCR, mixtures of synthesized cDNA, specific primers and a master mix (Maxima, SYBR Green/Fluorescein, Fermentas) were prepared according to the manufacturer's instructions. Measurements were done using a RotorGene 6000 instrument (Corbett), and the data were normalized to an endogenous control gene (HPRT). The relative mRNA expression levels were calculated based on the delta Ct method.

\section{Alkaline phosphatase activity measurement}

Levels of alkaline phosphatase activity were determined in cell lysates using $p$-nitrophenyl phosphate as the substrate (Anaspect). The procedure was carried out as recommended by the manufacturer. Briefly, cells growing in 24-well plates (NUNC, Wiesbaden, Germany) were washed with PBS and incubated with $1 \%$ Triton $\mathrm{X}-100$ for $30 \mathrm{~min}$ at $37{ }^{\circ} \mathrm{C}$. The resulting lysate was then incubated with the substrate and the amount of liberated $p$-nitrophenol ( $\mathrm{pNP}$ ) was measured at $405 \mathrm{~nm}$ (Genios plate reader, TECAN). Alkaline phosphatase activity values were normalized to the total protein content.

\section{Calcium content assay}

The amount of calcium minerals deposited on TCPS and the scaffolds by USSCs under osteogenic induction was measured using the cresolphthalein complex one method. Calcium extraction was performed by homogenization of the scaffolds in $0.6 \mathrm{~mol} / \mathrm{L} \mathrm{HCl}$ (Merck) followed by shaking for $4 \mathrm{~h}$ at $4{ }^{\circ} \mathrm{C}$. Optical density was measured at $570 \mathrm{~nm}$ after the addition of the reagent to the calcium solutions. Calcium content was obtained from a standard curve of concentrations of serial dilutions of calcium versus corresponding $O D$.

\section{Statistical analysis}

The Student's t-test was used to determine whether there is a significant difference between the groups. To compare the multiple groups, ANOVA test was applied; the difference was regarded as significant if the $P$ value was lower than 0.05 . Samples were run in triplicate for the biochemical assays and for molecular analysis, if not stated. All data are shown as means \pm standard deviation (SD).

\section{Results \\ Morphology and differentiation of USSCs}

The morphology of USSCs on TCPS was studied on $\mathrm{d} 3$ and d 21 of cultivation, with or without nHA suspension or osteogenic medium (Figure 1). According to our observations, in dense culture (21-d culture without any passage) USSCs proliferate and produce layers. Alizarin red staining of amorphous calcium deposits revealed strong mineralization in $21 \mathrm{~d}$ osteogenic medium induced USSCs while unstimulated USSCs did not show any calcium deposition on $\mathrm{d} 1$ (Figure 2). In this regard, in cells seeded on a scaffold, mineral deposition was noticeable with vividly porous structure composed from the aggregation of globular mineral accretions. Furthermore, PCR analysis of osteogenic marker genes, such as BMP2, ALPase, osteocalcin and osteopontin, confirmed osteogenic differentiation (Figure 3, Table 2). All of these marker genes showed low expression at $\mathrm{d} 1$ in unstimulated USSCs. The schematic
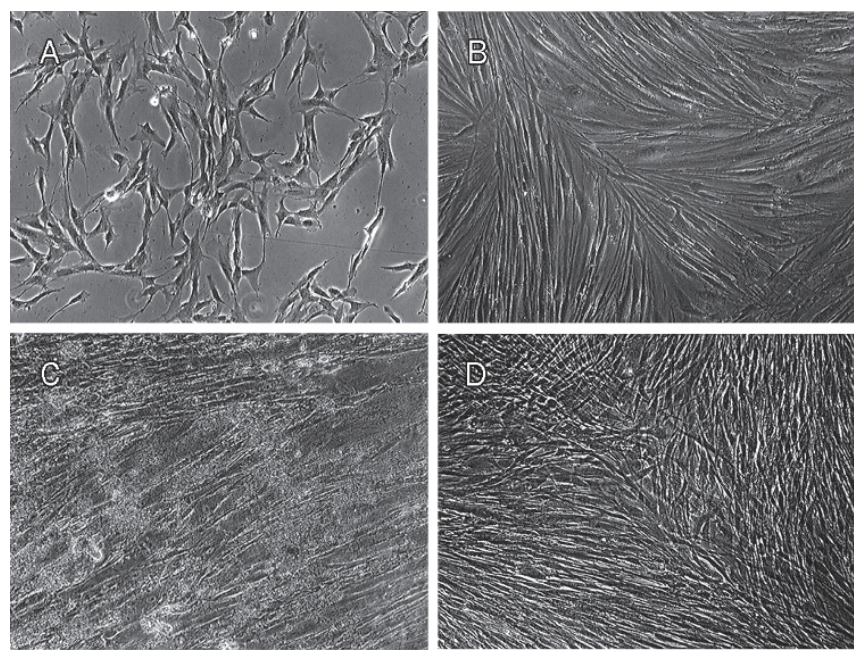

Figure 1. Phase microscopy for USSCs. Spindle-shaped USSCs on TCPS (A) on d 3, (B) Confluent multi-layer fibroblast-like shapes of USSCs on d 21 (self-differentiated), (C) in HA nanoparticle suspension and (D) in osteogenic medium (Bars: $100 \mu \mathrm{m}$. Magnification: $\times 40$ ). 


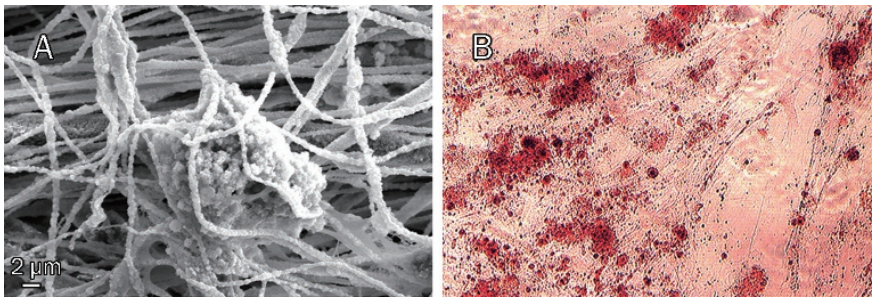

Figure 2. (A) Mineral deposition of USSC after $21 \mathrm{~d}$ of osteogenic differentiation on scaffold. (B) Alizarin red staining of calcium mineral deposited in the extracellular matrix after $21 \mathrm{~d}$ of osteogenic induction on TCPS.
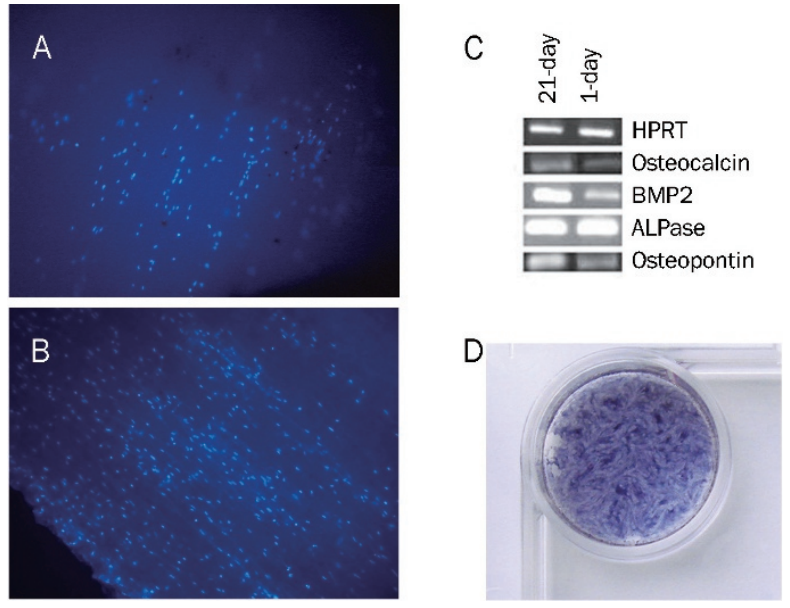

Figure 3. In order to show the high density and alignment of adherent cells on the scaffold, DAPI (4',6-diamidino-2-phenylindole dilactate, Invitrogen, CA, USA) was utilized for staining the nuclei of the fixed cultured cells $(0.25 \mathrm{~mL} /$ well of $1 \mu \mathrm{g} / \mathrm{mL}$ DAPI) after $1 \mathrm{~d}(\mathrm{~A})$ and $7 \mathrm{~d}(\mathrm{~B})$ for $30 \mathrm{~min}$ at room temperature. Photography was done with fluorescence microscope (Motic, Hong Kong, China) at $\times 100$ magnification. (C) Transcription of genes involved in osteogenic differentiation of USSCs. RT-PCR analysis of the expression of genes related to the osteogenic differentiation in 1-d and 21-d cultures on TCPS under osteogenic stimulation. Differentiated USSCs express the osteoblastic phenotype markers osteocalcin, osteopontin, BMP-2 and Alkaline phosphatase. HPRT was measured as internal control. (D) Staining USSCs to demonstrate multilayer proliferation after $21 \mathrm{~d}$ of culture by $500 \mu \mathrm{L}$ of sterile MTT dye (5 $\mathrm{mg} / \mathrm{mL}$, incubated for $4 \mathrm{~h}$ at $37^{\circ} \mathrm{C}$ ).

figure of expected multilayer growth of USSCs in nHA suspension culture is represented in Figure 4. Contrary to our expectation, apoptotic cells and cell debris were abundant in cultures containing the nHA suspension (Figure 1).

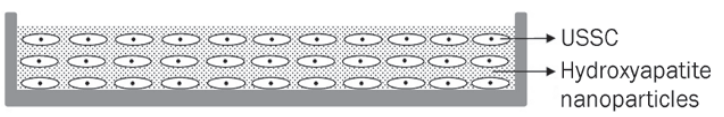

Figure 4. Schematic figure of trapped HA nanoparticle inside the USSCs layers.

\section{Characterization of nanofibers}

FTIR spectra of PCL/PLLA nanofibers, PCL/PLLA/nHA composite nanofibers, and nHA are shown in Figure 5. Characteristic peaks at 630 and $1016 \mathrm{~cm}^{-1}$ in the spectrum of nHA could be attributed to the vibrations of $\mathrm{PO}_{4}{ }^{3-}$ groups. In the PCL/PLLA/nHA composite scaffold spectrum, the latter peak overlapped with the vibration peak of PCL/PLLA at 1039 $\mathrm{cm}^{-1}$, which leads to a more intense peak in this region. For the PCL/PLLA/nHA scaffold, the vibration peak of nHA near $630 \mathrm{~cm}^{-1}$ could be confirmed and did not appear in the case of PCL/PLLA nanofibers.

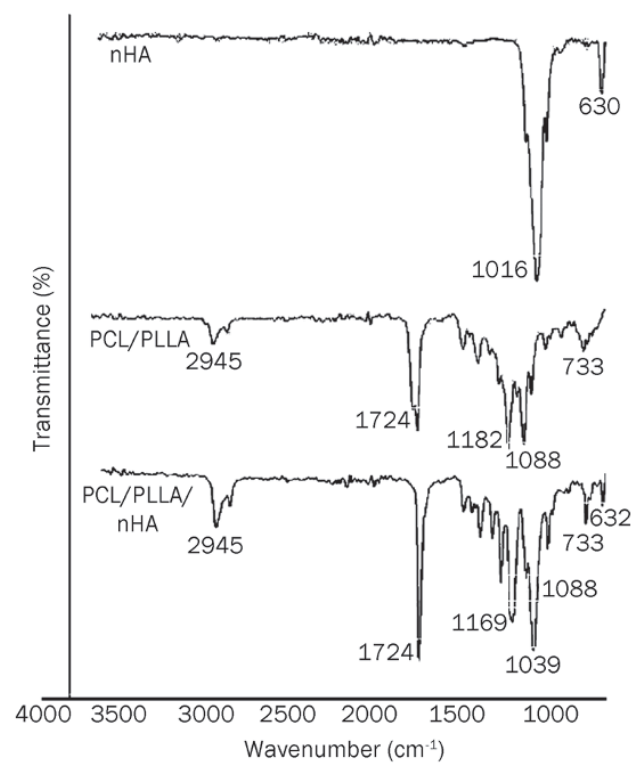

Figure 5. ATR-FTIR spectra of n-HA, PCL/PLLA, and PCL/PLLA/nHA.

PCL/PLLA nanofibers showed tensile strength of about $12 \mathrm{MPa}$ and elongation at break of $55 \%$, which decreased to a tensile strength of $4 \mathrm{MPa}$ and elongation at break of $38 \%$ after nHA was blended into the PCL/PLLA/nHA scaffolds (Figure 6).

Moreover, SEM micrographs (Figure 7) of the scaffold revealed an aligned morphology of porous, beadless and nano-scaled fibrous structures formed under controlled condi-

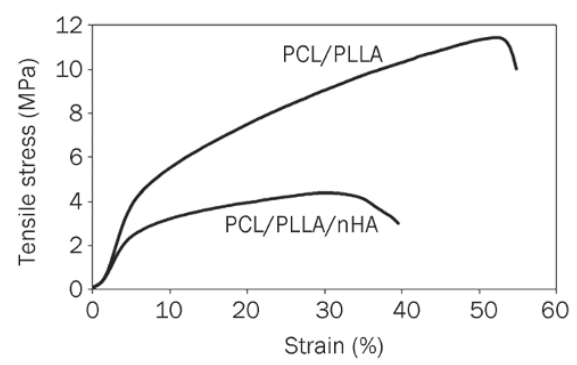

Figure 6. Stress-strain curves of aligned nanofibrous scaffolds along the fiber axis. 


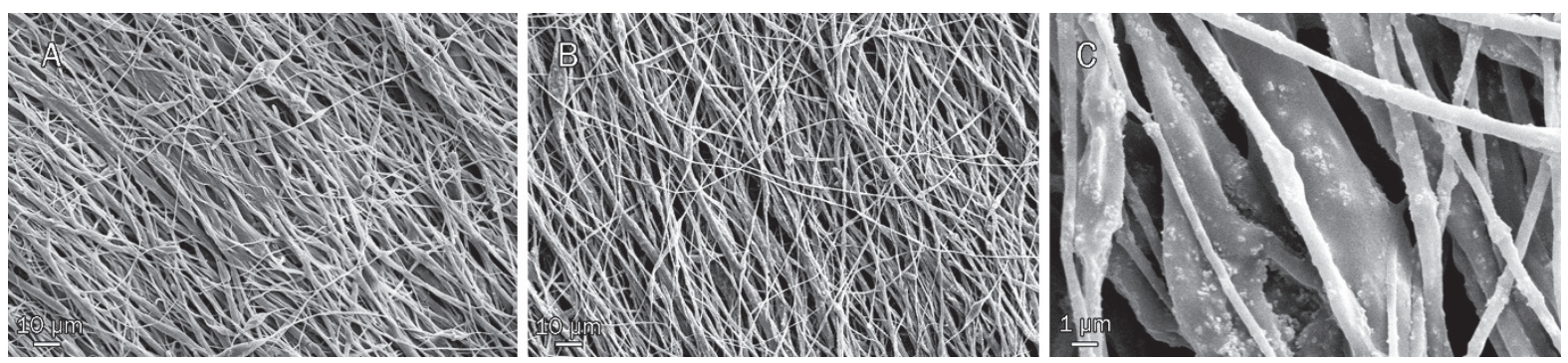

Figure 7. Morphology of fabricated scaffolds, PCL/PLLA (A, $\times 500)$, and PCL/PLLA/nHA $(B, \times 500)$ and $(C, \times 5000)$ nanofibers. As shown in the micrographs, electrospun scaffolds have a bead-free and oriented structure and HA nanoparticles are obvious within fibers. Thicker fibers are blends of PCL/HA and thinners are PLLA/HA.

tions. In addition, nHA appeared to be homogenously dispersed throughout the scaffolds.

\section{Morphology evaluation of seed cells on nanofibers}

The capacity of electrospun PCL/PLLA/nHA scaffolds to support the adhesion, proliferation and oriented growth of USSCs was investigated via nucleus staining of $1 \mathrm{~d}$ and $7 \mathrm{~d}$ cultures with DAPI (Figure 3). On d 1, the USSCs appeared to spread and adhere well, and on $\mathrm{d} 7$ the majority of the scaffold surface was covered by USSCs. In addition, the cells displayed oriented growth along the direction of fiber orientation on $\mathrm{d}$ 14, a finding that was also confirmed by SEM (Figure 8).
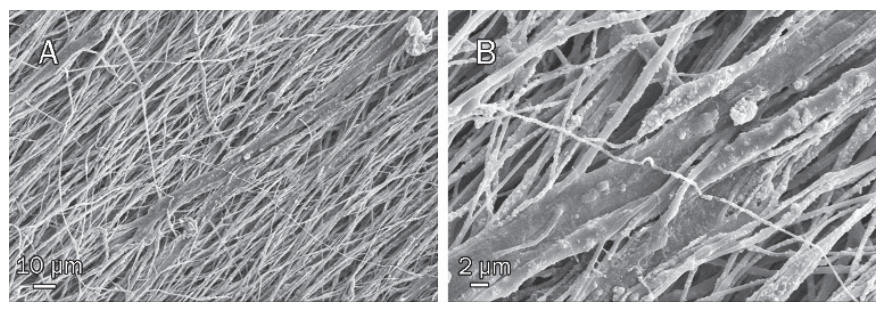

Figure 8. SEM micrographs of cell seeded nHA scaffolds after $14 \mathrm{~d}$ at $\times 500(A)$ and $\times 2000(B)$. It is obviously clear that the orientation of the cultured USSCs is aligned along the nanofibers.

\section{Cell proliferation}

We evaluated the effect of PCL/PLLA/nHA composite nanofibers and nHA suspension on the proliferation of USSCs by measuring the total protein contents of cultured USSCs in all mentioned groups on d 1, 7, 14, and 21 of cultivation (Figure 9). The total protein content at d 21 was significantly higher than that on $\mathrm{d} 7$ in all subgroups. Particularly in the TCPS and osteo-TCPS subgroups, the increase in total protein content between $\mathrm{d} 7$ and $\mathrm{d} 14$ was significant. In contrast, the total protein content of each subgroup from $\mathrm{d} 1$ to $\mathrm{d} 7 \mathrm{did}$ not change considerably. Furthermore, the rates of cell proliferation in the TCPS and osteo-TCPS subgroups were higher compared to the other groups. In addition, the rate of proliferation within group 2 (nHA and osteo-nHA subgroups) was less than that in the other groups.

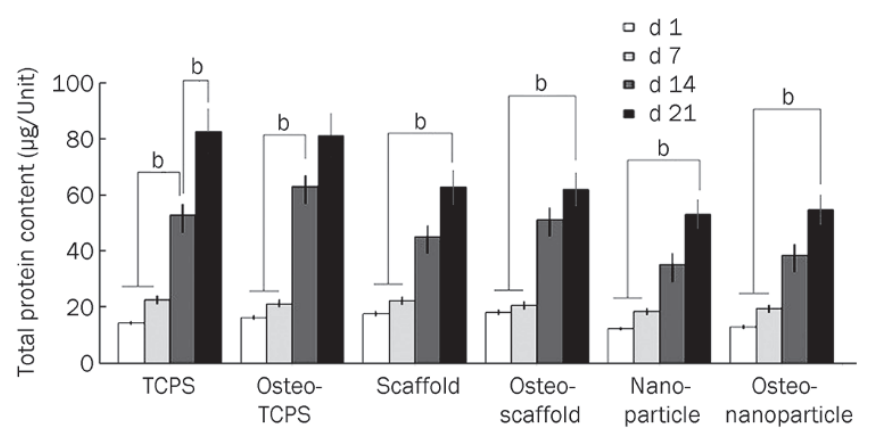

Figure 9. Comparison among total protein contents of USSCs on scaffolds and TCPS during osteogenic differentiation. ${ }^{\mathrm{b}} P<0.05$.

\section{ALPase activity measurement}

We analyzed ALPase activity to assess the progress of differentiation in all 6 subgroups (Figure 10). As illustrated, the highest ALPase activity belonged to the osteo-scaffold subgroup. Remarkably, the scaffold and osteo-scaffold subgroups demonstrated a decrease in ALPase activity on $\mathrm{d} 3$ when compared to $\mathrm{d} 1$ and $\mathrm{d} 7$; such a decrease was not observed in the other subgroups.

\section{Osteonectin transcription analysis}

We assessed osteonectin (ON) transcription quantitatively in all groups on $\mathrm{d} 7$ and $\mathrm{d} 21$. As shown in Figure 11, the scaffold

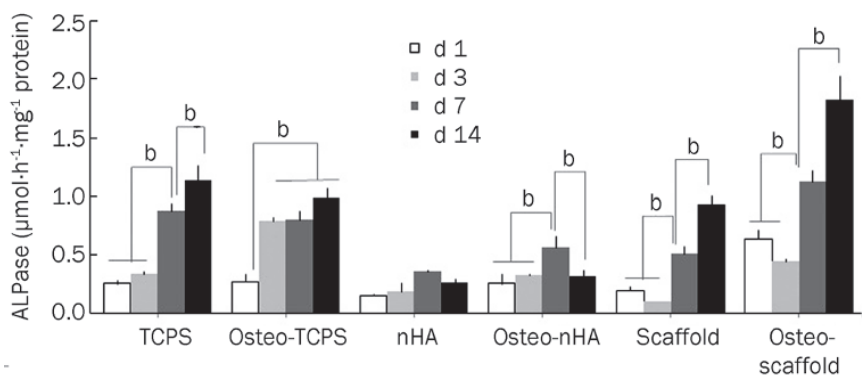

Figure 10. Measurement of alkaline phosphatase (ALPase) activity of USSCs after culturing for 1, 3, 7, 14, and $21 \mathrm{~d}$ in different conditions. Normalization is respect to total protein. It is clear that $d 14$ and osteoscaffold subgroup have the higher activity of ALPase. ${ }^{\mathrm{b}} P<0.05$. 
and osteo-scaffold subgroups demonstrated the highest levels on d 21. Conversely, the nHA and osteo-nHA subgroups transcribed osteonectin the least. Also, the transcription of osteonectin on d 21 was collectively higher than that on $\mathrm{d} 7$. Overall, similar increasing trends in $\mathrm{ON}$ transcription were observed for all groups.

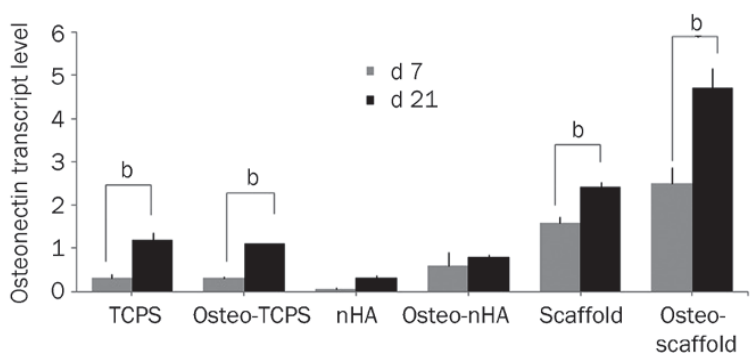

Figure 11. Relative expression of osteonectin on d 1, 3, 7, 14, and 21 in USSC on scaffolds and TCPS in different conditions. Normalization was done in respect to HPRT as an internal control. It is clear that osteoscaffold subgroup has the highest level of osteonectin transcript. In addition levels of osteonectin transcripts on d 21 is generally higher than d 7. ${ }^{\mathrm{b}} \mathrm{P}<0.05$.

\section{Calcium content quantity}

As the last osteogenic assessment, the calcium deposition (a late bone differentiation marker for the mineralization of USSCs) on d 21 of culture was examined and normalized to the total protein content (Figure 12). We know that calcium ions exist in the chemical structure of the nHA and HA scaffolds. To prevent this inherent calcium from introducing error into our measurements, we subtracted the amount of calcium deposited by the pure HA scaffold ( $6 \mu \mathrm{g} /$ scaffold) or nHA suspension $(10 \mu \mathrm{g} /$ well $)$ from related samples before normalization. The results indicate that calcium deposition increased significantly over time. In addition, the osteo-scaffold subgroup deposited the highest amount of calcium in comparison with the others, while the least calcium deposition was detected in the nHA subgroup.

\section{Discussion}

An important challenge in the regeneration of large bone

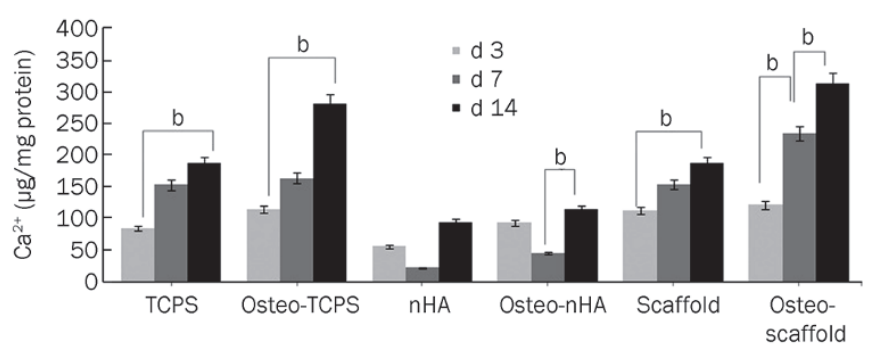

Figure 12. This figure represented the calcium content per total protein of different treatments of USSCs. It is obvious that d 14 has the highest content of calcium precipitate. Both osteo-induction and osteo-scaffold subgroups show the higher value for calcium precipitate. ${ }^{\mathrm{b}} P<0.05$. defects is a sufficient supply of tissue from suitable cells. In order to integrate hard tissue implants into defected bone, appropriate cells must bind to the implant matrices, differentiate, and form a new bone ${ }^{[37-39]}$. Hence, in this study we investigated both matrix combination and type of cell loaded into the matrix.

One of the most important aspects of bone tissue engineering is the introduction of appropriate cells into the matrices. In this regard, USSCs have unique biological characteristics and are able to undergo extensive replication in culture as well as differentiation under controlled conditions into multiple stromal lineages. USSCs can be considered a preferable alternative, allogenic source to MSCs for stem cell regeneration. Compared to MSCs, USSCs are easily accessible and routinely HLA matched after their isolation. Furthermore, their use does not raise ethical concerns ${ }^{[2,33,34]}$.

The differentiation potential of USSCs into the osteogenic lineage is well known and has been verified by many research groups $^{[36,40,41]}$. They can be differentiated into the osteogenic lineage both spontaneously and when cultured with specific supplements ${ }^{[36]}$. The control of osteogenic differentiation of USSCs is important for in vitro culture or delivery by matrices.

Although Kogler et al had declared in their primary publication in $2004^{[34]}$ that USSCs did not differentiate spontaneously, in $2009^{[36]}$ they stated that USSCs spontaneously differentiated into osteoblasts. The results of our study demonstrate that in dense culture ( $21 \mathrm{~d}$ culture without any passage) USSCs proliferate and they produce some layers (Figure 1). This high compactness facilitates their spontaneous differentiation into the osteogenic lineage. In 2004, Kogler et al ${ }^{[34]}$ had cultured USSCs to less than $80 \%$ confluency. Therefore, self-differentiation had not occurred. Also, Kogler et al ${ }^{[42]}$ reported that once USSCs started to develop into osteogenic cells, they failed at neural and endodermal differentiation. In another study, they showed that USSCs initiated bone formation in the orthotropic site $^{[34]}$.

Another research team has reported that USSCs were significantly engrafted into bone marrow, survived within a collagen-TCP scaffold for up to 4 weeks, and increased local bone formation in the femoral defect of a nude rat ${ }^{[2]}$. According to these reports and our prior observations about their osteogenic potential, using USSCs in bone tissue engineering appears to be appropriate. To examine this hypothesis, we analyzed some osteogenic markers over a period of $21 \mathrm{~d}$ in USSCs cultured in either growth medium (TCPS subgroup) or osteo-inductive supplements (osteo-TCPS subgroup).

For a closer look at the osteogenic behavior of USSCs, Alizarin red staining of amorphous calcium deposits (Figure 2) and the expression of osteogenic marker genes, such as BMP2, ALPase, osteocalcin and osteopontin, were analyzed in cells seeded on TCPS or scaffolds (Figure 3, Table 2). The results support the observation of osteogenic differentiation under stimulation. It is noteworthy that all of these marker genes showed little expression on d 1 (unstimulated USSCs). This result supports the osteoblastic potential of USSCs.

Handschel et $a l^{[43]}$ reported that the combination of insoluble 
collagenous bone matrix (ICBM) scaffolds and USSCs led to bone formation in immunocompromised rats. Nevertheless, the use of ICBM or any other donor-derived tissue or tissue portion raises the danger of immunological rejection. This risk makes the use of artificial matrices more appealing.

There are two main approaches to the design of support matrices. The first approach is the administration of large amounts of osteogenically predifferentiated cells in microsphere form ${ }^{[1,36]}$. But this technique is hampered by the limited volume of microspheres administered into the area of defect and the risk of effluence into other, unfavorable sites. Therefore, we have designed a new improvement in this field.

Because HA adsorbs adhesive proteins present within body fluids, HA containing scaffolds improve implant integration into bone defects. Many researchers have shown that protein adsorption is an important determinant of cell adhesion in early phases. Also, initial cell adhesion is critical for the functional improvement of anchorage-dependent cells because it mediates subsequent cellular responses, including proliferation and differentiation ${ }^{[3,44-47]}$.

In this study, our main objective was to evaluate the influence of nHA on osteogenic induction in USSCs. So, we chose TCPS as the reference surface for cultivation. Then, nHA grafted scaffolds and nHA suspensions were selected as two different approaches for the application of nHA and comparison for their osteogenic induction. This aim was based on a literature review of previous studies concerning applicable and suitable influences of HA in osteogenic induction.

In this regard, first the osteogenic influence of nHA particles in suspension was investigated (the sizes of the HA nanoparticles used were less than $200 \mathrm{~nm}$ ). This test was carried out because the osteogenic induction of nHA suspension has not been investigated in prior studies.

For the nHA suspension (group 2) approach, we added nHA once a week for 3 weeks into the culture plates of USSCs (Figure 4). We expected that proliferating USSCs retained these hard particles inside their layers, much like fillings in a sandwich. We had aimed to strengthen the USSCs multi-layer culture for use in bone regeneration. Practically, we achieved a transportable multi-layer of USSCs after $21 \mathrm{~d}$ culture, but it was not as suitable as we had anticipated. The cell population did not expand according to our prediction, which may be explained by the nHA-induced apoptosis.

In contrast to our expectation, we observed broad apoptosis of USSCs before suitable osteogenic differentiation in cultures treated with the nHA suspension. Yuan et al ${ }^{[48]}$ reported induction of apoptosis by nHA while analyzing the effects of different concentrations of HA nanoparticles on HepG2 cells. Chen et $a l^{[49]}$ reported that HA nanoparticle-induced apoptosis was mediated through the mitochondrial- and caspase-dependent pathway. In yet another study, Sun et al ${ }^{[50]}$ showed that HA nanoparticles could induce p53 expression; this activated downstream genes and finally resulted in cell apoptosis.

Considering that our observation is in agreement with the above-mentioned studies, it is feasible that the entry of USSCs into an apoptotic phase was induced by nHA. One explana- tion for this could be the fact that USSCs have to consume most of their cellular matter and energy to overcome the apoptotic phenomenon. Therefore, USSCs were forced to avoid apoptotic cascades before responding to growth signals or osteogenic induction. Our analyses on the expression of osteogenic markers and proliferation (total protein content) of USSCs under nHA suspension support our suggestion (Figure 9).

According to these findings, in our future research we propose the use of other concentrations of nHA suspensions or larger osteo-inductive particles, such as HA micro-rods, which cause no pinocytosis and consequent apoptosis in the USSCs.

Our second approach to matrix design is the fabrication of a nanofibrous scaffold. This method is considered a revolution in tissue engineering. Nanofibrous scaffolds loaded with the proper cells have been used in several studies, with various animal models and materials, ranging from chemical ceramics to biodegradable cages. These developments have resulted in a superior regeneration capacity for bioengineered bone compared to pure implants ${ }^{[37,38,51]}$.

The ability to mimic the chemical, physical and mechanical properties of the natural extra-cellular matrix is a key requirement for scaffolds to be successfully applied. Electrospun nanofibrous scaffolds have gained recognition in recent years because they are biomimetics of the nanoscale features of natural bone, such as high porosity, interconnected pores and large surface area ${ }^{[52]}$.

In this study, we successfully fabricated aligned electrospun PCL/PLLA/nHA nanofibrous scaffolds to evaluate the effects of grafted-nHA within biocomposite nanofibers on attachment, proliferation, and osteogenic differentiation of USSCs.

Analyses of FTIR spectra of PCL/PLLA nanofibers, PCL/ PLLA/nHA composite nanofibers and nHA indicated successful incorporation of nHA into the PCL/PLLA nanofibers (Figure 5). Incorporation of nHA into PCL/PLLA nanofibers causes a decrease in tensile strength and elongation at break. These changes occur because of low compatibility between nHA and these polymeric fibers. The large difference between the modulus of soft polymeric matrices and the modulus of hard nanoparticles leads to differences between the strains of these phases when tension is applied. Different strains and poor interfacial bonding between polymeric matrices and inorganic nHA lead to formation of voids at the interfaces. Growth of these voids leads to tearing of samples and decreased tensile properties of HA-filled nanofibers. Conversely, the large amount of inorganic nanoparticles $(20 \%$ of polymer weight $(w / w))$ resulted in agglomeration of filler particles, which caused some defects during application of tension. As a result, the PCL/PLLA/nHA nanofibrous scaffold was highly flexible and allowed USSCs to penetrate into the scaffolds. Characteristics such as a porous, beadless structure and nano-scale aligned fibers, as revealed by SEM micrographs make this scaffold suitable for tissue engineering applications. Moreover, nHA was effectively presented on the surface and helped to attain the rough surface morphology ideal for cell attachment and proliferation. 
Adherent cells convert signals sensed about matrix topography into morphological changes and cell-fate decisions concerning the direction of differentiation ${ }^{[53]}$. It has been suggested that the extracellular matrix and integrin collaborate to modulate both cell shape and intracellular signals ${ }^{[54,55]}$. However, the mechanisms by which topographical signals manage cell orientation and morphology are still poorly understood.

Findings on the effects of fiber orientation on cell morphology and growth have been published by various researchers. Because bone has significant anisotropic mechanical properties, with a highly oriented ECM and bone cells, the arrangement of the ECM mimicked by aligned nanofibrous scaffold is greatly preferred ${ }^{[56,57]}$.

In agreement with the findings of Yin et al ${ }^{[7]}$, aligned electrospun PLLA nanofibers provide an ideal microenvironment for hTSPCs to differentiate into the teno-lineage. An assay on electrospun polyester PCL-based polyurethane elastomer and PLLA demonstrated that alignment of electrospun fibers controlled the directional spreading of mouse fibroblasts ${ }^{[58]}$.

Other reports have investigated the effect of oriented PCL electrospun fibers on promoting Schwann cell maturation, astrocyte alignment and directed axonal outgrowth for encouraging neural repair in the future ${ }^{[59,60]}$. Finally, yet another study reported that unidirectionally oriented nanofibers significantly induced muscle cell alignment and myotube formation compared to randomly oriented nanofibers ${ }^{[61]}$.

Cell infiltration into an electrospun nanofibrous matrix is often limited due to the relatively small pore size between the fibers. Aligned nanofibers provide relatively suitable pore size so that cell infiltration into the nanofibrous matrices can be significantly enhanced. The effect of aligned nanofibers on promoting infiltration of endothelial cells into scaffolds was reported by Kurpinski et al ${ }^{[62]}$. The present study investigated whether aligned nanofibers influenced the infiltration of USSCs and the orientation of their differentiation.

The impact of fabricated scaffolds on cell adhesion, growth and production of an aligned morphology of seeded USSCs on nanofibers was investigated by nucleus staining and SEM micrographs (Figures 3 and 8 ). Our results suggest that this scaffold is a biocompatible matrix. Such aligned scaffolds may lead to the development of desirable engineered bony tissue composed of favorable stem cells and smart scaffolds that can organize and align cell populations to facilitate osteogenic differentiation.

The consequence of our chosen conditions on cell proliferation was investigated by measuring the total protein content of cultured USSCs. Because we had used the same initial number of cells for all groups, we can consider the "total protein content" an indicator of proliferation. All groups showed an increase in total protein content during differentiation (Figure 9). The most important reason for the reduction in proliferation rate (decrease in total protein content) of two group (nHA and osteo-nHA subgroups) could be attributed to apoptosis induced by HA nanoparticles.

Compared to TCPS, the nHA scaffold demonstrated a decreased capacity to support proliferation of USSCs. To explain this insignificant difference, we should consider the modifications to the scaffold surface; further improvement may be required. Collectively, our findings showed biocompatibility and the capacity of this scaffold to support the proliferation of USSCs over a 21-d period of monitoring.

High-level expression of certain genes, which occurs at discrete time points during the differentiation process, is thought to contribute to the differentiated state of osteoblasts. Alkaline phosphatase (ALPase), collagen 1 (Col I) and osteonectin (ON) are expressed at high levels near the end of the proliferative period and during the period of extracellular matrix deposition and maturation. Genes expressed at or near the time of mineralization include osteopontin and osteocalcin ${ }^{[63]}$.

Measuring the ALPase activity, as an indicator of progress in osteogenic differentiation, showed the highest levels of this factor at d 14 (Figure 10). Similar results were obtained by Jones et $a l^{[64]}$, where the ALPase activity for human osteoblast cells (HOB) was highest on d 14, after which a decrease was observed due to the beginning of mineralization in HOB. In an experiment by Babister et $a l^{[1]}$, both the activity and the transcription of ALPase in hMSCs were highest on d 21. Conversely, Degistirici et $a l^{[41]}$ found that USSCs expressed the most active ALPase on $\mathrm{d} 7$ during osteogenic differentiation.

Based on the fact that USSCs tend to differentiate into the osteo-lineage potentially, we can assume that USSCs passaged for longer will express osteogenic markers sooner. Consequently, the USSC line used by Degistirici et a ${ }^{[41]}$ may have been older than ours.

To explain the decrease of ALPase activity on d 3, when compared to $\mathrm{d} 1$ and $\mathrm{d} 7$ in the scaffold group, it is worth mentioning that USSCs are adherent cells. Therefore, the properties of the matrix affect its function and destination. After seeding on a scaffold, USSCs need some time to adapt to the new matrix. As a consequence, seeded USSCs on HA scaffolds show a delay in responding to environmental cues. The similarity in the ALPase activity in the TCPS and osteo-TCPS subgroups could be another strong piece of evidence for the hypothesis that USSCs tend to spontaneously differentiate into the osteo-lineage.

The results of the ALPase activity assay were further confirmed by qPCR analysis of osteonectin and calcium content analysis in all conditions (Figures 11 and 12). Similar increasing trends in $\mathrm{ON}$ transcription were observed in all groups, with maximum transcription on $\mathrm{d} 21$. As the final osteogenic evaluation, calcium deposition was examined and normalized to the total protein contents. In accordance with the previous results, calcium deposition increased significantly over time. Considering all the results, seeding USSCs on PCL/PLLA/ nHA nanofibers synergistically improved the ALPase activity, osteonectin transcription and calcium deposition of USSCs in osteogenic culture conditions.

\section{Conclusion}

In the present study, nHA in both aligned electrospun nanofibrous PCL/PLLA scaffolds and in suspension was investigated to determine the most effective condition for the control 
of osteogenic differentiation and mineralization of USSCs. SEM images indicated homogenous dispersion of nHA throughout this multi-component scaffold and aligned growth of USSCs on the scaffold. The incorporation of HA into the scaffold had no negative impact on USSC initial adhesion, proliferation, spreading or viability.

In conclusion, relying on these data, the described scaffold, in combination with an osteogenic medium, enhances osteogenesis in USSCs more effectively than the other analyzed conditions. These enhancements were confirmed by biochemical and gene expression analysis. Therefore the synergism of USSCs and the PCL/PLLA/nHA scaffold can be used to fill up spaces in bony defects and is a promising candidate for a novel bone regeneration approach.

\section{Acknowledgements}

This work was supported financially by the Stem Cell Technology Research Center, National Elite Foundation and Iran Nanotechnology Initiative Council (INIC).

\section{Author contribution}

Behnaz BAKHSHANDEH designed and performed the research, Masoud SOLEIMANI designed the research, and Nasser GHAEMI and Iman SHABANI contributed new reagents or analytic tools.

\section{References}

1 Babister JC, Hails LA, Oreffo RO, Davis SA, Mann S. The effect of pre-coating human bone marrow stromal cells with hydroxyapatite/ amino acid nanoconjugates on osteogenesis. Biomaterials 2009; 30: 3174-82.

2 Jager M, Degistirici O, Knipper A, Fischer J, Sager M, Krauspe R. Bone healing and migration of cord blood-derived stem cells into a critical size femoral defect after xenotransplantation. J Bone Miner Res 2007; 22: 1224-33.

3 Lee JH, Rim NG, Jung HS, Shin H. Control of osteogenic differentiation and mineralization of human mesenchymal stem cells on composite nanofibers containing poly[lactic-co-(glycolic acid)] and hydroxyapatite. Macromol Biosci 2010; 10: 173-82.

4 Yang F, Murugan R, Wang S, Ramakrishna S. Electrospinning of nano/micro scale poly(L-lactic acid) aligned fibers and their potential in neural tissue engineering. Biomaterials 2005; 26: 2603-10.

5 Shor L, Guceri S, Wen X, Gandhi M, Sun W. Fabrication of threedimensional polycaprolactone/hydroxyapatite tissue scaffolds and osteoblast-scaffold interactions in vitro. Biomaterials 2007; 28: 5291-7.

6 Jose MV, Thomas V, Xu Y, Bellis S, Nyairo E, Dean D. Aligned bioactive multi-component nanofibrous nanocomposite scaffolds for bone tissue engineering. Macromol Biosci 2010; 10: 433-44.

7 Yin Z, Chen X, Chen JL, Shen WL, Hieu Nguyen TM, Gao L, et al. The regulation of tendon stem cell differentiation by the alignment of nanofibers. Biomaterials 2010; 31: 2163-75.

8 Ge Z, Yang F, Goh JC, Ramakrishna S, Lee EH. Biomaterials and scaffolds for ligament tissue engineering. J Biomed Mater Res A 2006; 77: 639-52.

9 Hashemi SM, Soleimani M, Zargarian SS, Haddadi-AsI V, Ahmadbeigi $\mathrm{N}$, Soudi S, et al. In vitro differentiation of human cord bloodderived unrestricted somatic stem cells into hepatocyte-like cells on poly(epsilon-caprolactone) nanofiber scaffolds. Cells Tissues Organs
2009; 190: 135-49.

10 Vasita R, Katti DS. Nanofibers and their applications in tissue engineering. Int J Nanomedicine 2006; 1: 15-30.

11 Gupta D, Venugopal J, Mitra S, Giri Dev VR, Ramakrishna S. Nanostructured biocomposite substrates by electrospinning and electrospraying for the mineralization of osteoblasts. Biomaterials 2009; 30: 2085-94.

12 Li LH, Kommareddy KP, Pilz C, Zhou CR, Fratzl P, Manjubala I. In vitro bioactivity of bioresorbable porous polymeric scaffolds incorporating hydroxyapatite microspheres. Acta Biomater 2010; 6: 2525-31.

13 Yang X, Yang F, Walboomers XF, Bian Z, Fan M, Jansen JA. The performance of dental pulp stem cells on nanofibrous $\mathrm{PCL} /$ gelatin/ nHA scaffolds. J Biomed Mater Res A 2010; 93: 247-57.

14 Liu X, Li X, Fan Y, Zhang G, Li D, Dong W, et al. Repairing goat tibia segmental bone defect using scaffold cultured with mesenchymal stem cells. J Biomed Mater Res B Appl Biomater 2010; 94: 44-52.

15 Niu X, Feng Q, Wang M, Guo X, Zheng Q. Porous nano-HA/collagen/ PLLA scaffold containing chitosan microspheres for controlled delivery of synthetic peptide derived from BMP-2. J Control Release 2009; 134: 111-7.

16 Ramay HR, Zhang M. Biphasic calcium phosphate nanocomposite porous scaffolds for load-bearing bone tissue engineering. Biomaterials 2004; 25: 5171-80.

17 Wang M. Developing bioactive composite materials for tissue replacement. Biomaterials 2003; 24: 2133-51.

18 Marletta G, Ciapetti G, Satriano C, Pagani S, Baldini N. The effect of irradiation modification and RGD sequence adsorption on the response of human osteoblasts to polycaprolactone. Biomaterials 2005; 26: 4793-804.

19 Yu H, Wooley PH, Yang SY. Biocompatibility of Poly-epsilon-caprolactone-hydroxyapatite composite on mouse bone marrow-derived osteoblasts and endothelial cells. J Orthop Surg Res 2009; 4: 5.

20 Yoshimoto H, Shin YM, Terai H, Vacanti JP. A biodegradable nanofiber scaffold by electrospinning and its potential for bone tissue engineering. Biomaterials 2003; 24: 2077-82.

21 Shin $\mathrm{M}$, Yoshimoto $\mathrm{H}$, Vacanti JP. In vivo bone tissue engineering using mesenchymal stem cells on a novel electrospun nanofibrous scaffold. Tissue Eng 2004; 10: 33-41.

22 Wright-Charlesworth DD, King JA, Miller DM, Lim CH. In vitro flexural properties of hydroxyapatite and self-reinforced poly(L-lactic acid). J Biomed Mater Res A 2006; 78: 541-9.

23 Woo KM, Seo J, Zhang R, Ma PX. Suppression of apoptosis by enhanced protein adsorption on polymer/hydroxyapatite composite scaffolds. Biomaterials 2007; 28: 2622-30.

24 Wang X, Song G, Lou T. Fabrication and characterization of nanocomposite scaffold of PLLA/silane modified hydroxyapatite. Med Eng Phys 2010; 32: 391-7.

25 Hunt JA, Callaghan JT. Polymer-hydroxyapatite composite versus polymer interference screws in anterior cruciate ligament reconstruction in a large animal model. Knee Surg Sports Traumatol Arthrosc 2008; 16: 655-60.

26 Sun JJ, Bae CJ, Koh YH, Kim HE, Kim HW. Fabrication of hydroxyapatite-poly(epsilon-caprolactone) scaffolds by a combination of the extrusion and bi-axial lamination processes. J Mater Sci Mater Med 2007; 18: 1017-23.

27 Charles LF, Shaw MT, Olson JR, Wei M. Fabrication and mechanical properties of PLLA/PCL/HA composites via a biomimetic, dip coating, and hot compression procedure. J Mater Sci Mater Med 2010; 21 : 1845-54.

28 Ngiam M, Liao S, Patil AJ, Cheng Z, Yang F, Gubler MJ, et al. Fabrication of mineralized polymeric nanofibrous composites for bone graft materials. Tissue Eng Part A 2009; 15: 535-46. 
46 Wilson CJ, Clegg RE, Leavesley DI, Pearcy MJ. Mediation of biomaterial-cell interactions by adsorbed proteins: a review. Tissue Eng 2005; 11: 1-18.

47 Romberger DJ. Fibronectin. Int J Biochem Cell Biol 1997; 29: 93943.

48 Yuan Y, Liu C, Qian J, Wang J, Zhang Y. Size-mediated cytotoxicity and apoptosis of hydroxyapatite nanoparticles in human hepatoma HepG2 cells. Biomaterials 2010; 31: 730-40.

49 Chen S, Zhao Y, Han W, Zhao G, Zhu L, Wang J, et al. Mitochondriadependent signalling pathway are involved in the early process of radiation-induced bystander effects. Br J Cancer 2008; 98: 183944.

50 Sun J, Ding T. p53 reaction to apoptosis induced by hydroxyapatite nanoparticles in rat macrophages. J Biomed Mater Res A 2009; 88: 673-9.

51 Kon E, Muraglia A, Corsi A, Bianco P, Marcacci M, Martin I, et al. Autologous bone marrow stromal cells loaded onto porous hydroxyapatite ceramic accelerate bone repair in critical-size defects of sheep long bones. J Biomed Mater Res 2000; 49: 328-37.

52 Murugan R, Ramakrishna S. Design strategies of tissue engineering scaffolds with controlled fiber orientation. Tissue Eng 2007; 13: 1845-66.

53 Gao L, McBeath R, Chen CS. Stem cell shape regulates a chondrogenic versus myogenic fate through Rac1 and N-cadherin. Stem Cells 2010; 28: 564-72.

54 Engler AJ, Sen S, Sweeney HL, Discher DE. Matrix elasticity directs stem cell lineage specification. Cell 2006; 126: 677-89.

55 Boudreau NJ, Jones PL. Extracellular matrix and integrin signalling: the shape of things to come. Biochem J 1999; 339: 481-8.

56 Li WJ, Mauck RL, Cooper JA, Yuan X, Tuan RS. Engineering controllable anisotropy in electrospun biodegradable nanofibrous scaffolds for musculoskeletal tissue engineering. J Biomech 2007; 40: 168693.

57 Zhu B, Lu Q, Yin J, Hu J, Wang Z. Alignment of osteoblast-like cells and cell-produced collagen matrix induced by nanogrooves. Tissue Eng 2005; 11: 825-34.

58 Truong YB, Glattauer V, Lang G, Hands K, Kyratzis IL, Werkmeister JA, et al. A comparison of the effects of fibre alignment of smooth and textured fibres in electrospun membranes on fibroblast cell adhesion. Biomed Mater 2010; 5: 25005.

59 Horne MK, Nisbet DR, Forsythe JS, Parish C. Three dimensional nanofibrous scaffolds incorporating immobilized BDNF promote proliferation and differentiation of cortical neural stem cells. Stem Cells Dev 2010; 19: 843-52.

60 Nisbet DR, Rodda AE, Horne MK, Forsythe JS, Finkelstein DI. Neurite infiltration and cellular response to electrospun polycaprolactone scaffolds implanted into the brain. Biomaterials 2009; 30: 4573-80.

61 Choi JS, Lee SJ, Christ GJ, Atala A, Yoo JJ. The influence of electrospun aligned poly(epsilon-caprolactone)/collagen nanofiber meshes on the formation of self-aligned skeletal muscle myotubes. Biomaterials 2008; 29: 2899-906.

62 Kurpinski KT, Stephenson JT, Janairo RR, Lee H, Li S. The effect of fiber alignment and heparin coating on cell infiltration into nanofibrous PLLA scaffolds. Biomaterials 2010; 31: 3536-42.

63 Beck GR Jr, Zerler B, Moran E. Phosphate is a specific signal for induction of osteopontin gene expression. Proc Natl Acad Sci U S A 2000; 97: 8352-7.

64 Jones JR, Tsigkou O, Coates EE, Stevens MM, Polak JM, Hench LL. Extracellular matrix formation and mineralization on a phosphate-free porous bioactive glass scaffold using primary human osteoblast (HOB) cells. Biomaterials 2007; 28: 1653-63. 